\title{
DESEMPENHO PSICOLINGUÍSTICO E ESCOLAR DE IRMÃOS COM MIELOMENINGOCELE
}

\section{Psycholinguistic and school performance of brothers with myelomeningocele}

\author{
Dionísia Aparecida Cusin Lamônica ${ }^{(1)}$, Amanda Tragueta Ferreira (2), \\ Lívia Maria do Prado ${ }^{(3)}$, Patrícia de Abreu Pinheiro Crenitte ${ }^{(4)}$
}

\begin{abstract}
RESUMO
Tema: mielomeningocele e consequências para o desenvolvimento de habilidades psicolinguísticas e escolares. Procedimentos: o objetivo deste estudo foi descrever e refletir sobre o desempenho psicolinguístico e escolar de irmãos com mielomeningocele lombar baixa. Participaram do estudo um menino de 14 anos e 5 meses (P1) e sua irmã de 6 anos e 6 meses (P2). Os procedimentos constaram de entrevista com familiares, Observação do Comportamento Comunicativo (OCC), Teste de Vocabulário por Imagens Peabody - TVIP, Teste Illinóis de Habilidades Psicolinguísticas (ITPA), Perfil de Habilidades Fonológicas (PHF), Teste de Desempenho Escolar (TDE), e Reconhecimento de letras e números (RLN). A análise dos resultados foi descritiva respeitando os manuais dos instrumentos. Resultados: a linguagem oral encontra-se sem alteração evidente. No ITPA e PHF, verificou-se que P1 e P2 apresentaram dificuldade nas habilidades de memória, closura auditiva, expressão verbal, combinação de sons e consciência fonológica. P1 ainda apresentou dificuldade na habilidade de recepção visual e P2 nas habilidades de associação auditiva e closura gramatical. Ambas as crianças apresentaram alteração nas atividades de aprendizagem. Conclusão: os participantes do estudo apresentaram comportamento comunicativo, habilidades de linguagem oral e vocabulário receptivo sem alterações evidentes. P1 e P2 apresentaram alterações nas habilidades psicolinguísticas bem como no desempenho de atividades envolvendo leitura, escrita e aritmética. As dificuldades apresentadas causam impacto importante nas atividades acadêmicas, demonstrando vulnerabilidade cerebral no sistema de suporte para atividade de aprendizagem.
\end{abstract}

DESCRITORES: Meningomielocele; Avaliação de Desempenho; Desenvolvimento da Linguagem; Aprendizagem

(1) Fonoaudióloga. Doutora em Distúrbios da Comunicação Humana pela Universidade Federal de São Paulo. LivreDocente. Professora Associada do Departamento de Fonoaudiologia da Faculdade de Odontologia de Bauru da Universidade de São Paulo.

(2) Fonoaudióloga. Mestranda em Fonoaudiologia pela Faculdade de Odontologia de Bauru da Universidade de São Paulo.

(3) Fonoaudióloga. Mestranda em Fonoaudiologia pela Faculdade de Odontologia de Bauru da Universidade de São Paulo.

(4) Fonoaudióloga. Doutora em Ciências Médicas pela Universidade Estadual de Campinas (UNICAMP). Professora Doutora do Departamento de Fonoaudiologia da Faculdade de Odontologia de Bauru da Universidade de São Paulo.

Conflito de interesses: inexistente

\section{INTRODUÇÃO}

A mielomeningocele (MMC) é um dos defeitos de fechamento do tubo neural que pode acarretar dificuldades urológicas, ortopédicas, neurológicas, gastrointestinais, psicossociais e intelectuais ${ }^{1-4}$. Estes indivíduos apresentam forte potencial para desenvolver problemas de aprendizagem associados à presença de alterações no desenvolvimento motor, linguístico, cognitivo e no processamento das informações ${ }^{5-11}$. Tendem a apresentar nível intelectual variável, na dependência das anormalidades neuropatológicas, das anomalias da neuroembriogênese, das complicações decorrentes 
do tratamento cirúrgico, da hidrocefalia, da estimulação ambiental, dentre outros ${ }^{1,2,7,12}$. Com isto, são indivíduos de risco para desenvolver problemas de aprendizagem associados à redução do funcionamento cognitivo, déficits perceptivos e alteração nas habilidades não verbais , $^{7,9,10,12-14}$.

Quanto ao desempenho de linguagem, estas crianças podem apresentar alterações em todos os níveis linguísticos ${ }^{15}$, entretanto, a expectativa é para alterações nas habilidades não verbais com envolvimento da organização visuo-espacial, dos processos perceptivos, do controle de atenção, da discriminação, da memória seletiva e, especialmente, das tarefas que envolvam aprendizagem seriada $^{9-11,14-18}$, influenciando negativamente no aprendizado da leitura, escrita e matemática ${ }^{7,8,11,13-18}$.

Diante do exposto, o objetivo deste estudo foi descrever e refletir sobre o desempenho psicolinguístico e escolar de irmãos com mielomeningocele.

\section{APRESENTAÇÃO DO CASO}

Este estudo foi encaminhado para o Comitê de Ética em Pesquisa da Faculdade de Odontologia de Bauru da Universidade de São Paulo e aprovado (protocolo 011/2010). A participação dos sujeitos ocorreu com a assinatura do Termo de Consentimento Livre e Esclarecido pelo representante legal dos participantes, seguindo a Resolução 196/96 do CONEP, permitindo a divulgação e estudo dos resultados da avaliação os participantes em estudos desta natureza.

Participaram do estudo um menino de 14 anos e 5 meses de idade (P1) e uma menina de 6 anos e 6 meses (P2), irmãos, filhos de pais não consanguíneos, ambos com diagnóstico de mielomeningocele lombar baixa ao nascimento, vindos à Clínica de Fonoaudiologia com a seguinte queixa "Eles falam bem, mas estamos preocupados com as dificuldades que estão apresentando para o aprendizado escolar".

Os procedimentos aplicados constaram de entrevista detalhada com os familiares, Observação do Comportamento Comunicativo (OCC), Teste de Vocabulário por Imagens Peabody - TVIP ${ }^{19}$, Teste Illinóis de Habilidades Psicolinguísticas (ITPA) ${ }^{20}$, Perfil de Habilidades Fonológicas (PHF) ${ }^{21}$, Teste de Desempenho Escolar (TDE) ${ }^{22}$, e Reconhecimento de letras e números (RLN). A análise dos resultados foi realizada conforme descrito nos manuais dos instrumentos aplicados e será apresentada de maneira descritiva.

Na Figura 1 estão descritos os dados referentes à história pregressa e desenvolvimento de $\mathrm{P} 1$ e P2, coletados durante a entrevista

\section{RESULTADOS}

$\mathrm{Na}$ OCC verificou-se que P1 e P2 apresentaram intenção comunicativa com interação satisfatória. Quanto às habilidades expressivas conversacionais observou-se em P1 e P2 produção de turnos ocupados com enunciados extensos e coerentes, sempre contextualizados. No discurso narrativo pode-se observar uso de pronomes pessoais, nome de objetos cotidianos, lugares, verbos e atributos com sentido lexical, bem como, pronomes possessivos, advérbios de espaço e advérbios de tempo com significado gramatical. O discurso narrativo de P1 e P2 é elaborado com frases encadeadas, sem comprometimento de coerência e coesão. Os participantes demonstraram compreensão adequada de contextos concretos e abstratos referentes às situações imediatas e mediatas.

$\mathrm{Na}$ Tabela 1, estão descritos os resultados do Teste de Vocabulário por Imagem Peabody, Teste Illinóis de Habilidades Psicolinguísticas, Perfil de Habilidades Fonológicas, Teste de Desempenho Escolar e Reconhecimento de letras e números.

\section{DISCUSSÃO}

A Figura 1 refere os resultados obtidos na entrevista na qual verificou-se a ocorrência de MMC em irmãos ambos com lesão em nível lombar baixa. Dentre os defeitos de fechamento do tubo neural a MMC é a mais frequente, afetando cerca de $85 \%$ do total de casos. De etiologia desconhecida, mas com características multifatoriais (genéticas e ambientais), sua incidência mundial é variável, sendo em média de 1 para 1000 nascidos vivos com chance de recorrência de $5 \%$ para o segundo filho ${ }^{3,4}$.

O tipo de MMC lombar baixa representa comprometimentos musculares de adutores, quadríceps, flexores mediais do joelho, e eventualmente tibial anterior e/ou glúteo médio, que explicam as alterações de marcha e os achados de mau funcionamento da bexiga ${ }^{3}$ e dificuldades para controle de esfíncteres, confirmando informações descritas pelos familiares quanto a estes aspectos. Com relação às correções cirúrgicas da MMC e hidrocefalia, P1 realizou a cirurgia aos 30 dias e aos 10 meses e P2 aos 2 dias e 20 dias respectivamente. Centros internacionais especializados no tratamento dos defeitos do tubo neural consideram que a cirurgia da MMC deve ocorrer até as primeiras 72 horas de vida, para evitar o aumento das complicações $^{3}$ e que estes indivíduos podem ter associada a hidrocefalia, com necessidade de procedimento cirúrgico para instalação de derivações ventriculares. Estudos revelam que crianças com MMC e hidrocefalia têm demonstrado significantes 
Tabela 1 - Resultados do TVIP, ITPA, PHF, TDE e RLN

\begin{tabular}{llcc}
\hline & Instrumento & P1 (14a 5m) & P2 (6a 6m) \\
\hline TVIP & & Média alta & Média \\
\hline & Recepção auditiva & $10 \mathrm{a} 11 \mathrm{~m}$ & $7 \mathrm{a}$ \\
& Recepção visual & $6 \mathrm{a}$ & $10 \mathrm{a} 11 \mathrm{~m}$ \\
& Associação auditiva & $10 \mathrm{a} 11 \mathrm{~m}$ & $4 \mathrm{a} 3 \mathrm{~m}$ \\
& Associação visual & $10 \mathrm{a} 11 \mathrm{~m}$ & $6 \mathrm{a} 6 \mathrm{~m}$ \\
& Memória Auditiva & $9 \mathrm{a} 6 \mathrm{~m}$ & $2 \mathrm{a} 10 \mathrm{~m}$ \\
ITPA $^{*}$ & Memória visual & $10 \mathrm{a} 11 \mathrm{~m}$ & $8 \mathrm{a}$ \\
& Closura auditiva & $8 \mathrm{a} 3 \mathrm{~m}$ & $6 \mathrm{a}$ \\
& Closura visual & $10 \mathrm{a} 11 \mathrm{~m}$ & $10 \mathrm{a} 11 \mathrm{~m}$ \\
& Closura gramatical & $10 \mathrm{a} 6 \mathrm{~m}$ & $4 \mathrm{a}$ \\
& Expressão verbal & $10 \mathrm{a}$ & $4 \mathrm{a} 4 \mathrm{~m}$ \\
& Expressão manual & $10 \mathrm{a} 11 \mathrm{~m}$ & $10 \mathrm{a} 11 \mathrm{~m}$ \\
& Combinação de sons & 8a $6 \mathrm{~m}$ & $2 \mathrm{a}$ \\
\hline \multirow{2}{*}{ PHF } & & Desempenho esperado para & Desempenho sob atenção \\
& & crianças de 10 anos & para crianças de 5 anos \\
\hline TDE & Escore Total & Classificação inferior & Classificação inferior \\
\hline \multirow{2}{*}{ RLN } & & Reconhecimento total & Reconhece poucas letras e \\
& & & números \\
\hline
\end{tabular}

${ }^{*}$ A idade máxima do teste é 10 anos e 11 meses.

\begin{tabular}{|c|c|c|}
\hline ENTREVISTA & P1 & $\mathbf{P 2}$ \\
\hline Gestação e Parto & Sem intercorrências & Sem intercorrências \\
\hline Diagnóstico MMC & ao nascimento & ao nascimento \\
\hline Fala & 12 meses & 12 meses \\
\hline Marcha & 36 meses $^{*}$ & 24 meses $^{*}$ \\
\hline Controle esfíncter & Ausente & Ausente \\
\hline Saúde Geral & $\begin{array}{l}30 \text { dias: cirurgia da MMC lombo-sacral } \\
6 \text { meses: refluxo gastroesofágico } \\
10 \text { meses: válvula derivação } \\
\text { (hidrocefalia) } \\
10 \text { e } 14 \text { anos: cirurgia pés } \\
\text { Frequentes Infecções de urina Miopia }\end{array}$ & $\begin{array}{l}2 \text { dias: cirurgia da MMC lombo-sacral } \\
20 \text { dias: válvula derivação (hidrocefalia) } \\
3 \text { e } 4 \text { anos: cirurgia pés } \\
\text { Frequentes Infecções de urina }\end{array}$ \\
\hline Reabilitação & $\begin{array}{l}\text { Fisioterapia } \\
\text { Terapia Ocupacional }\end{array}$ & $\begin{array}{l}\text { Fisioterapia } \\
\text { Terapia Ocupacional }\end{array}$ \\
\hline Escola & $\begin{array}{l}\text { Regular } \\
7^{\text {a }} \text { série } \\
\text { Frequentes mudanças de professores } \\
\text { (4 vezes) } \\
\text { Queixa de dificuldades na } \\
\text { aprendizagem }\end{array}$ & $\begin{array}{l}\text { Regular } \\
\text { EMEI (pré-escola) } \\
\text { Queixa de dificuldades na } \\
\text { aprendizagem }\end{array}$ \\
\hline
\end{tabular}

* Com auxílio. Vale ressaltar que, atualmente, P1 e P2 fazem uso de órteses e auxílio de muleta na realização da marcha.

Figura 1 - Dados da entrevista 
diferenças individuais no funcionamento neurológico e comportamental ${ }^{2,8,9,11,12,18}$, reforçando a necessidade das correções cirúrgicas o mais precocemente possível para evitar os efeitos deletérios para o funcionamento neurológico e comportamental. Tais diferenças, segundo os autores, são reflexos da combinação de influências neurológicas, médicas, familiais, ambientais as quais essas crianças estão expostas.

O desenvolvimento da linguagem tem caráter multifatorial e apesar dos fatores de risco envolvidos na $\mathrm{MMC}$, relacionados à influência do SNC no desempenho global, neuropsicomotor, perceptivos e da cognição somados aos períodos frequentes de hospitalização e aos aspectos psicossociais quanto a estigma e superproteção, as habilidades não verbais são mais comprometidas nestes indivíduos $^{5,6,10}$ do que as habilidades verbais. Tais aspectos foram verificados na OCC, pois $\mathrm{P} 1$ e P2 não apresentaram dificuldades relacionadas ao comportamento comunicativo e uso da linguagem no diálogo. Ressalta-se que na conversação informal, não são exigidas habilidades específicas e mesmo com alterações morfossintáticas na expressão verbal, o conteúdo pode ser transmitido de maneira adequada, com a possibilidade da manutenção do uso da linguagem no diálogo e compreensão dos conteúdos linguísticos por interlocutores. Estes achados são relatados em muitos estudos que abordam esta temática ${ }^{5,6,9,10,14,17}$.

Os participantes do estudo apresentaram classificação média para suas idades cronológicas referente ao vocabulário receptivo (Tabela 1). Crianças com MMC podem apresentar alterações nos diversos níveis linguísticos, entretanto a linguagem oral pode estar aparentemente normal e a expectativa é para alterações nas habilidades não verbais ${ }^{5,6,9-11,14-18}$, com influência nas atividades de aprendizagem da leitura e escrita, este aspecto explicaria a conduta familiar de procura do fonoaudiólogo somente na idade escolar.

Nos procedimentos ITPA e PHF (Tabela 1), verificou-se que $\mathrm{P} 1$ e $\mathrm{P} 2$ apresentaram dificuldade no desempenho das habilidades de memória e closura auditiva, expressão verbal, combinação de sons e consciência fonológica. P1 ainda apresentou dificuldade na habilidade de recepção visual e P2 nas habilidades de associação auditiva e closura gramatical.

Cabe ressaltar, que P1 apresentou escores compatíveis com a idade máxima do teste para as habilidades de recepção e associação auditiva, associação, memória e closura visual e expressão manual. Entretanto, a idade cronológica deste participante é superior à idade máxima de avaliação do ITPA. Mesmo sabendo desta circunstância, a aplicação deste instrumento foi válida, uma vez que este é composto por diferentes provas que compõe o rol de habilidades psicolinguísticas necessárias ao processo de aprendizagem escolar. Diante disso, é de grande valia a análise geral do desempenho do $\mathrm{P} 1$, somadas às queixas familiares quanto às atividades acadêmicas, além da análise observacional dos avaliadores durante a realização das provas. Desta forma, considerando o grau de escolaridade e a idade cronológica de $\mathrm{P} 1$, verifica-se que há exigências psicolinguísticas que este participante não consegue cumprir, o que nos permite inferir que existe também alteração nestas habilidades avaliadas no ITPA, nas quais ele obteve pontuação referente a 10 anos e 11 meses, idade máxima do teste.

Os participantes apresentaram déficits em habilidades auditivas que se referem à capacidade de reproduzir memorizando uma sequência de dígitos apresentada auditivamente (memória auditiva), completar partes ausentes de uma palavra captada por meio da apresentação auditiva (closura auditiva) e relacionar conceitos apresentados oralmente por meio de analogias verbais (associação auditiva). P2 apresentou de maneira evidente maior dificuldade nas habilidades auditivas quando comparadas às visuais. A literatura apresenta que indivíduos com mielomeningocele formam um grupo heterogêneo, quanto ao seu desempenho nas habilidades verbais e não verbais, que tendem a ter comprometimento em graus variáveis nos aspectos envolvendo o controle da atenção, discriminação, memória seletiva tanto auditiva quanto visual, interferindo sobremaneira nas tarefas de aprendizagem seriada, resolução de problemas e rendimento acadêmico ${ }^{15,23}$.

Estas habilidades são pré-requisito para compreensão e execução de ordens complexas, bem como para as habilidades de aprendizagem². Há relatos da literatura ${ }^{24}$ reportando que as crianças com MMC tendem a apresentar dificuldades perceptuais envolvendo relações de categorias, analogias, associações e coordenação das representações perceptuais relacionadas.

Tanto $P 1$ quanto $P 2$ apresentaram alteração para expressar conceitos verbais como classe ou denominação, forma, composição, função, partes principais, quantificação, comparação (expressão verbal) e para utilizar a redundância da linguagem oral na aquisição da sintaxe e inflexões gramaticais, o que engloba o conhecimento de aspectos morfológicos tais como flexões de gênero, número e grau, formas verbais de modo, tempo, pessoa e voz e aspectos sintáticos da utilização de conectivos, pronomes e outros (closura gramatical). Estes dados são condizentes com a literatura quando refere que estas 
crianças apresentam as habilidades linguísticas básicas frequentemente intactas, porém com falhas em habilidades discursivas envolvendo a flexibilidade no uso da linguagem $2,9,12,24,25$.

A habilidade que P1 apresentou maior dificuldade foi recepção visual que engloba a capacidade de extrair significados a partir de símbolos de natureza visual. Embora o participante apresente alteração da acuidade visual (miopia), infere-se que não houve influência da mesma no resultado do subteste, uma vez que P1 faz uso de lentes corretivas. Estudos apresentam que indivíduos com MMC demonstram alterações nas habilidades de percepção visual e também no processamento de interações e associações visuais ${ }^{24}$.

Verificou-se que a habilidade mais defasada da P2 é a combinação de sons, também apresentada com déficit por $\mathrm{P} 1$. Esta habilidade refere-se à integração dos processos associativos, mais especificamente quanto à síntese fonêmica, uma das habilidades envolvidas na prova de consciência fonológica (PHF), na qual P1 e P2 apresentaram escore abaixo do esperado para a idade cronológica. Estudos recentes demonstram que crianças com dificuldade de aprendizagem podem apresentar alterações nas habilidades fonológicas e no acesso ao léxico mental, decorrentes de modificações em diferentes níveis do processamento da informação $0^{8,26,27 . ~}$

No TDE (Tabela 1) P1 e P2 apresentaram desempenho inferior ao esperado para a série escolar que frequentam. P2 conseguiu escrever apenas seu primeiro nome e não foi capaz de realizar a leitura de nenhuma palavra. Diante disto, aplicou-se a prova de reconhecimento de letras e números (Tabela 1) em P2. Nesta prova observouse que reconheceu poucas letras e apresentou dificuldade em produzir oralmente o alfabeto.

O aprendizado da escrita engloba diferenciar os traçados das letras, saber a que sons as letras correspondem, estabelecer correspondências quantitativas, identificar a posição da letra dentro da palavra, compreender que uma mesma letra pode representar vários sons, assim como um mesmo som pode ser representado por diversas letras ${ }^{27}$. É possível pressupor que a compreensão do princípio alfabético está baseada nos seguintes fatores facilitadores: capacidade de segmentar a língua falada em unidades distintas, consciência de que estas unidades repetem-se em diferentes palavras faladas e conhecimento das regras de correspondência entre grafemas e fonemas ${ }^{25}$. Estas dificuldades foram observadas nos resultados obtidos pelos participantes nas provas de consciência fonológica do PHF e no subteste combinação de sons do ITPA. Diversos fatores influenciam na aquisição e desenvolvimento da aprendizagem, como o ambiente familiar ${ }^{14}$, o desenvolvimento da linguagem oral e também das habilidades perceptuais que irão favorecer a apreensão das estratégias utilizadas no processo de aprendizagem ${ }^{7,9,10,14}$.

A literatura apresenta que crianças com MMC são de risco para distúrbio de aprendizagem, o qual está associado ao funcionamento cognitivo, percep-

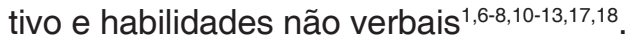

P1 apresentou maior prejuízo nas atividades que envolviam aritmética do que nas atividades que envolviam habilidades de leitura e escrita, apesar de também comprometidas. Já P2 não realizou nenhum exercício de aritmética bem como apresentou dificuldade no reconhecimento dos números.

Crianças com dificuldades na decodificação da leitura e dificuldade na matemática têm demonstrado déficits em memória de trabalho verbal, visual e processamento fonológico, experimentando prejuízos para a resolução de problemas matemáticos, na decodificação dos conceitos e interpretação dos enunciados, bem como na estimativa numérica ${ }^{15,16}$.

As alterações nas habilidades psicolinguísticas apresentadas pelos participantes certamente influenciaram diretamente no desempenho das tarefas envolvendo leitura, escrita e aritmética, o que pode explicar a queixa dos pais quanto à aprendizagem e rendimento escolar dos filhos. Tais dificuldades são descritas na literatura ${ }^{5-8,10,12,13,15,23,26,27}$, quanto às dificuldades de aprendizagem da leitura e escrita de indivíduos com mielomeningocele. Entretanto, cabe ressaltar, que como as habilidades linguísticas básicas são desenvolvidas sem grandes dificuldades ${ }^{23}$ nestes indivíduos, e estão frequentemente intactas do ponto de vista de comunicação interpessoal há uma tendência das dificuldades linguísticas somente serem identificadas no período da aprendizagem escolar, quando são identificadas as dificuldades no processo de aquisição da linguagem escrita. Com isto, sugere-se que estas crianças sejam acompanhadas, quanto ao desenvolvimento das habilidades comunicativas ainda no período pré-escolar para que seja possível realizar processo de intervenção visando minimizar a influência negativa das alterações nas habilidades perceptuais e psicolinguísticas para a aquisição da leitura e escrita, possibilitando o desenvolvimento do real potencial da criança, bem como menor prejuízo nas habilidades escolares.

\section{CONCLUSÃO}

Os participantes do estudo apresentaram comportamento comunicativo, habilidades de linguagem oral e vocabulário receptivo sem 
alterações evidentes. Quanto às habilidades psicolinguísticas, verificaram-se alterações importantes que refletiram no desempenho das habilidades de leitura, escrita, e aritmética contribuindo para interferência negativa no processo de aprendizagem escolar de ambos os irmãos. As dificuldades apresentadas causam impacto importante nas atividades acadêmicas, demonstrando vulnerabilidade cerebral no sistema de suporte para atividade de aprendizagem.

\section{ABSTRACT}

Background: meningomyelocele and consequences on the development of psycholinguistic and school abilities. Procedures: the purpose of this study was to describe and reflect on the psycholinguistic and school performance of siblings with low lumbar myelomeningocele. The study included a boy, 14 years and 5 months (P1) and his sister, 6 years and 6 months (P2). The procedures consisted of an interview, Communicative Behavior Observation (CBO), Vocabulary Test Peabody Picture - PPVT, Illinois Test of Psycholinguistic Abilities (ITPA), Phonological Abilities Profile (PAP), School Performance Test (SPT), and recognition of letters and numbers (RLN). The analysis was descriptive and respecting the manuals of the instruments. Results: the oral language has no obvious change. In ITPA and PAP, we found that P1 and P2 had difficulty in memory skills, closure listening, verbal, combination of sounds and phonological awareness. P1 still had difficulty in the ability of visual reception and P2 in the abilities of auditory association and closure grammar. Both children showed difficulties in the learning activities. Conclusion: the study subjects performed quite well in communicative skills, oral language and receptive vocabulary without obvious changes. P1 and P2 had changes in psycholinguistic skills and performance of activities involving reading, writing and arithmetic. Difficulties cause major impact on academic activities, demonstrating the vulnerability of the brain in the system supporting learning activity.

KEYWORDS: Meningomyelocele; Employee Performance Appraisal; Language Development; Learning

\section{REFERÊNCIAS}

1. Barf HA, Verhoef M, Jennekens-Schinkela. G, Gooskens RHJM, Prevo AJH. Cognitive status of young adult with spina bifida. Dev Med Child Neurol. 2003; 45:813-20.

2. Lindquist B, Uvebrant $P$, Rehn E, Carlsson G. Cognitive function in children with myelomeningocele without hydrocephalus. Childs Nerv Syst. August 2009; 25(8), online.

3. Iglesias $\mathrm{J}$, Ingilde $M$, Naddeo $S$, Sánchez $M$, Spinelli S, Van der Velde J, Yánez A. Deteccion e tratamiento del mielomeningocele por um equipo interdisciplinario. Rev Hosp Mat Inf Ramón Sarda. 2000; 19:11-7.

4. Fernandes AC. Malformações do tubo neural. In: Hebert S, Xavier R, Pardini Jr AG, Barros Filho TEP. Ortopedia e traumatologia: princípios e prática. 3 ed. Porto Alegre: Artmed; 2003. p. 839-57.

5. Fletcher JM, Barners M, Dennis M. Language Development in Children With Spina Bifida. Seminars in Pediatric Neurology. 2002; 9(3):201-8.
6 Russell C. Understanding Nonverbal Learning Disorders in Children With Spina Bifida. Teaching Exceptional Children. 2004; 36(4):8-13.

7. Vachha, B; Adams, R. C. Memory and selective learning in children with spina bifida mielomeningocele and shunted hydrocephalus: A preliminary study. http.//creativecommons.org/ licence/by/2.0 November, 2005

8. Barnes MA, Dennis M, Hetherington R. Reading and writing skills in young adults with spina bifida and hydrocephalus. J Int Neuropysychol Soc. 2004; 10:655-63.

9. Lomax-Bream LE, Barnes M, Copeland K, Taylor HB, Landry SH. The impact of spina bifida on development across the first 3 years. Develop Neuropsychol, 2007; 31(1):1-20.

10. Ris MD, Ammerman RT, Waller N, Walz N, Openheimer S, Brown TM, Enrile BG, Yates KO. Toxanocity of nonverbal learning disabilities in spina bifida. J Int Neuropsychol Soc. 2007; 13:50-8.

11. Swartwout MD, Cirino PT, Hampson AW, Fletcher JM, Brandt ME, Dennis M. Sustained 
attention in children with two etiologies of early hydrocephalus. Neuropsychol, 2008; 22(6):765-75. 12. Lindquist $B$, Persson EK, Uverant $P$, Carlsson G. Learning memory and executive functions in children with hydrocephalus. Acta Paediatric, 2008; 97(5):591-601.

13. Lindquist B, Carlsson G, Persson EK, Uverant $P$. Learning disabilities in a population-based group children with hydrocephalus. Acta Paedriatric. 2005; 94(7): 877-83.

14. Vachha B, Adams R. Implications of family environment and language development: comparing typically developing children to those with spina bífida. Child: care, health and develop. 2009; 35(5):709-16.

15. Barnes MA, Wilkinson $M$, Khemani $E$, Boudesquie A, Dennis M, Fletcher JM. Arithmetic Processing in Children with Spina Bifida: calculation accuracy, strategy use, and fact retrieval fluency. J Learn Dis. 2006; 39(2):174-87.

16. English LH, Barnes MA, Taylor HB, Landry SH. Mathematical Development in Spina Bifida. Develop Dis Res Rev. 2009; 15:28-34.

17. Dennis $M$, Jewell $D$, Hetherington R, Burton $C$, Brandt ME, Blaser SE, Fletcher JM. Verb generation in children with spina bifida. J Int Neuropsychol Soc. 2008; 14:181-91.

18. Sawin KJ, Joy P, Bakker K, Shores EA, West C. Object-based visual processing in children with spina bifida and hydrocephalus: a cognitive neuropsychological analysis. J Neuropsychol. 2009; 3:229-44.

19. Dunn LM, Padilla ER, Lugo DE, Dunn LM. Teste de Vocabulário em Imagens Peabody (Peabody Picture Vocabulary Test), adaptação Hispanoamericana. Espanha: Circle Pines: American Guidance Service, 1986.
20. Bogossian MADS. Teste de Illinois de habilidades psicolinguística: crítica do modelo mediacional e de diversos aspectos da validade do instrumento. Rio de Janeiro; 1984. [Tese de Doutorado, Fundação Getúlio Vargas].

21. Alvarez AMMA, Carvalho IAM, Caetano AL. Perfil de Habilidades Fonológicas: manual. São Paulo: Via Lettera Editora e Livraria Ltda. $2^{\underline{a}}$ edição, 2004.

22.Stein LM. TDE: teste de desempenho escolar: manual para aplicação e interpretação. São Paulo: Casa do Psicólogo, 1994.

23. Boyer KM, Yates KO, Enrile BG. Working memory and information processing speed in children with mielomeningocele and shunted hydrocephalus: Analysis of the Children's paced auditory serial addition test. J Int Neuropsychol Society. 2006; 12;305-13.

24. Dennis M, Landry SH, Barnes M, Fletcher JM. A modelo $f$ neurocognitive function in spina bífida over the life span. J Int Neuropsychol Soc. 2006; 12:825-96.

25. Guimarães SRK. O aperfeiçoamento da concepção alfabética de escrita: relação entre consciência fonológica e representações ortográficas. In: Maluf MR. Metalinguagem e aquisição da escrita. São Paulo: Casa do Psicólogo; 2003. p. 149-84.

26. Dias RS, Ávila CRB. Uso e conhecimento ortográfico no transtorno específico da leitura. Rev Soc Brás Fonoaudiol. 2008; 13(4):381-90.

27. Capellini AS, Conrado TLBC. Desempenho de escolares com e sem dificuldades de aprendizagem de ensino particular em habilidade fonológica, nomeação rápida, leitura e escrita. Rev CEFAC. 2009; 11(2):183-93.
http://dx.doi.org/10.1590/S1516-18462011005000103

RECEBIDO EM: 23/03/2010

ACEITO EM: 20/07/2010

Endereço para correspondência:

Dionísia Aparecida Cusin Lamônica

Departamento de Fonoaudiologia da Faculdade

de Odontologia USP

Al. Octávio Pinheiro Brisolla 9-75

Bauru - SP

CEP: 17053-095

E-mail: dionelam@uol.com.br 